Conclusion In conclusion, under the conditions of this study, low-energy laser stimulation plus exercise did not provide a significant advantage over exercise alone.

\section{FRI0237 ASSESSMENT OF A FUNCTIONAL RESTORATION PROGRAM FOR PATIENTS WITH CHRONIC LOW BACK PAIN}

G Casso, C Cachin, JC Gerster. Service d'Anesthésiologie, Centre Hospitalier Universitaire Vaudois, Lausanne Et Hôpital d'Orbe, Orbe, Switzerland

\subsection{6/annrheumdis-2001.541}

\section{Background}

Objectives The aim of this open study was to evaluate the effectiveness of a functional restoration program for patients suffering from chronic disabling low back pain. Return to work 12 months after the end of the program was chosen as the main outcome.

Methods A questionnaire was sent to 125 patients $(115 \mathrm{M}, 10 \mathrm{~F}$, mean age 40 years). The mean duration of absence from work before entry in the program was 4 months. All patients had followed an identical functional restoration program in a hospital setting 12 months before. The program included $6 \mathrm{~h}$ of physical therapy and ergonomics, 5 days per week for 3 weeks.

Results 109 questionnaires could be assessed. 57 patients (52\%) were back to work, 39 (36\%) full time and $18(17 \%)$ at half time. 42 patients were not working. A shorter duration out of work before entry in the program, an improvement of the isokinetic trunk strength at the end of the program, a positive self assessment of the program and the absence of psychiatric comorbidity were all separate predictors of a favourable outcome.

Conclusion Our results are in agreement with those of the European literature. An intensive restoration functional program had positive effects in $53 \%$ of our selected population.

\section{FRI0238 EFFICACY AND SAFETY OF LOXOPROFEN IN ACUTE LOW BACK PAIN}

J Natour, E Puertas, A Radu, M Freire, R Bonfiglioli. Rheumatology, Federal University of São Paulo, São Paulo, Brazil

\subsection{6/annrheumdis-2001.542}

Background Nonsteroidal anti-inflammatory drugs (NSAIDs) are the most frequent and effective prescribed medication to treat patients with low back pain (LBP) but are associated with a high incidence of GI adverse events. Loxoprofen sodium, a NSAID prodrug from phenylpropionic group, claimed to have lesser GI and renal toxicity, has a large clinical experience in Japan, but its use in occidental people is scarce. Thus, it was conducted a clinical trial with Loxoprofen in the treatment of acute LBP in comparison with diclofenac $\mathrm{K}$, using a validated assessment questionnaire for monitoring the outcome.

Objectives Objective of this double blind, randomised, controlled, multicentre study was compare efficacy and safety of Loxoprofen with that of diclofenac in the treatment of acute LBP.

Methods Patients of both gender, aged $>18$ years, with non-specific lumbar pain complying with exclusion criteria were admitted to the study. After the informed consent, patients were randomly allocated into the treatment groups - Loxoprofen 60 mg t.i.d. (LOX) or diclofenac $50 \mathrm{mg}$ t.i.d. (DIC). Duration of treatment was 2 weeks. Patients were evaluated at pre-treatment and weekly during therapy for the following parameters: overall severity of symptoms; pain at rest, on pressure, and on movement using a visual analogue scale (VAS); physical examination (Schober and lateral flexion indexes, finger-floor distance); physician and patient global impression; side-effects; and Roland-Morris Questionnaire for LBP duly validated to Brazilian patients.

Results A total of 96 patients were treated in 5 centres ? 47 with LOX (19 $\mathrm{M}$ and $28 \mathrm{~F}$ ), and 49 with DIC (16 $\mathrm{M}$ and $33 \mathrm{~F}$ ). There was no significant difference between groups. $83 \%$ of cases were classified as acute in LOX; in DIC, $57 \%$ of cases were acute, $29 \%$ were sub acute and $14 \%$ recurrent. Severity of symptoms, pain (at rest, on movement, and on pressure), and objectives indexes showed statistically significant improvements compared with baseline ( $p<0.001$ ? chi square) with both treatments; there were no statistically significance differences between groups. Roland-Morris Questionnaire showed a good correlation with evolution of symptoms. 14 patients in LOX reported adverse events, being 12 GI effects (25.5\%), and 23 patients in DIC, with 18 GI effects $(36.7 \%)$ ( $p<0.001)$. Epigastralgia was the most prevalent GI event reported. Four patients in LOX and 7 in DIC discontinued therapy due to GI adverse events. Tolerability assessments were classified as both very good and good in $97 \%$ in LOX and in $90 \%$ in DIC ( $p<0.05$ ). Physician and patient overall assessment were $>95 \%$ as either very good or good in both groups (n.s.).

Conclusion Loxoprofen $60 \mathrm{mg}$, t.i.d., showed efficacy and excellent tolerability and safety in acute low back pain, superior to that of an established NSAID, diclofenac $50 \mathrm{mg}$, t.i.d. RollandMorris Questionnaire was a useful tool to assess the disease outcome.

\section{FRI0239 PAIN THERAPY OF FAILED BACK SURGERY SYNDROME PATIENTS (FBSS): SUCCESSFUL LONG-TERM TREATMENT WITH FENTANYL TTS}

${ }^{1} \mathrm{H}$ Haentzschel, ${ }^{2} \mathrm{~S}$ Schwalen, ${ }^{3} \mathrm{~J}$ Kraemer, ${ }^{3} \mathrm{~T}$ Theodoridis. ${ }^{1}$ Medizinische Klinik Und Poliklinik IV, University of Leipzig, Leipzig; ${ }^{2}$ Medical and Scientific Affairs, Janssen-Cilag GmbH, Neuss; ${ }^{3}$ Orthopaedic Department, University of Bochum, Bochum, Germany

10.1136/annrheumdis-2001.543

Background Patients with a FBSS suffer from chronic severe back pain. Most of these patients have had a postlaminectomy in the lumbosacral region. 10\% of patients with a postlaminectomy develop chronic pain after the surgery. The percentage of positive outcomes after a re-surgery amount about $30-75 \%$. In general the outcome of these patients is unfavourable. At present pain therapy with opioids is not generally recommended in patients with FBSS.

Objectives Documentation of long-term efficacy and safety of fentanyl TTS treatment for patients who have had at least one surgical intervention for chronic back pain and who continue to suffer from severe back pain.

Methods Subgroup analysis of an open, international, multicentre study (FEN-INT-13) with a duration of 12 months. 85 patients (mean age 50 years; 46 women; mean pain duration 9 years) with failed back surgery syndrome received fentanyl TTS in an initial dosage of equivalent analgesic strength to prior opioid therapy. The dose was subsequently adjusted to provide optimum pain therapy. Visits have been scheduled day 1 , week 1, month 1-12. The primary target parameter was the subjective weekly assessment of pain control (very good, good, moderate, 
poor, very poor). In addition the treatment preference with regard to the prior analgesic treatment and the quality of life (SF-36) has been documented. Regarding the safety unexpected events and vital parameters have been documented.

Results $59 \%$ of the patients were treated for 12 months, $41 \%$ discontinued treatment earlier $(5 \%$ because of inadequate effect, the rest for other reasons). The primary target parameter of subjective weekly assessment of pain control was consistently rated very good to moderate (50 to $80 \%$ ). $77 \%$ of patients preferred fentanyl TTS to the former opioid therapy. There was a significant improvement in SF-36 (quality of life) after 12 months in terms of physical pain, mental health, average pain intensity and pain frequency. The mean fentanyl TTS dose was $67 \mu \mathrm{g} / \mathrm{h}$ in month $1,90 \mu \mathrm{g} / \mathrm{h}$ in months 6 and $97 \mu \mathrm{g} / \mathrm{h}$ in month 12 . The most frequently reported adverse reactions caused by fentanyl TTS (>10\%) were nausea, vomiting, headache, increased perspiration, constipation, drowsiness, fatigue, dizziness and depression.

Conclusion In this trial patients with chronic, therapy-resistant back pain showed benefit of a treatment with fentanyl TTS. There was not only pain reduction but also improvement in quality of life over a period of one year. At the same time the dosage of fentanyl remained stable. The success of this pain therapy may offer the possibility to initiate a causal therapy like physiotherapy. Fentanyl TTS offer stable pain control for failed back surgery syndrome patients and improves their quality of life. It is safe and well tolerated.

\section{FRI0240 LASER NUCLEOLYSIS FOR THE TREATMENT OF LOMBOSCIATICA. RESULTS IN A COHORT OF 30 PATIENTS WITH A 6 MONTHS FOLLOW UP}

${ }^{1} \mathrm{R}$ Dupuis, ${ }^{2} \mathrm{~B}$ Lavignolles, ${ }^{2} \mathrm{~L}$ Salanon, JR Vigne ${ }^{3},{ }^{3} \mathrm{D}$ Liguoro, ${ }^{3} \mathrm{~F}$ San Galli, ${ }^{3} \mathrm{M}$ Dautheribes, ${ }^{1} \mathrm{~J}$ Dehais, ${ }^{3} \mathrm{~J}$ Guérin. ${ }^{1}$ Rheumatology; ${ }^{2}$ Orthopaedic Surgery; ${ }^{3}$ Neurosurgery, CHU Bordeaux, Bordeaux, France

\subsection{6/annrheumdis-2001.544}

Background Since 1986, laser is used for percutaneous treatment of discal herniation. Due to a thermic effect, it infers an evaporation of the nucleus pulposus and allows a discal decompression that seems to be responsible for the improvement of the patients.

Objectives To estimate the efficacy of the laser nucleolysis on pain, functional incapacity and quality of life of patients suffering of lombosciatica due to dics herniation.

Methods Thirty patients suffering from lombosciatica due to a disc herniation for more than three months were selected on clinical and radiological criteria. All were not relieved although they underwent a well-driven medical treatment. They were treated with percutaneous way with a laser diode $940 \mathrm{~nm}$. All were followed prospectively for six months by an examiner different from the operator. Evaluation criteria consisted of level of lumbar and radicular pain (VAS), level of functional incapacity (Oswestry) and quality of life (SF-36).

Results 30 patients were included in this study. 6 months after the intervention 23 of them $(76.6 \%)$ were relieved of their radicular pain (EVA $<20 \%$ ). Lumbar pain and level of functional incapacity decreased whereas the quality of life increased. The patients who failed to improve three months after the procedure (23.4\%) were all relieved later after a surgical discectomy.

Conclusion Laser nucleolysis allows a significant decrease at month 6 of pain, functional incapacity and an increase of the quality of life in a group of selected patients suffering from lombosciatica resistant to the medical treatment.

\section{FRI0241 LUMBAR LORDOSIS IN ACUTE AND CHRONIC LOW BACK PAIN PATIENTS}

${ }^{1} \mathrm{D}$ Evcik, ${ }^{2} \mathrm{~A}$ Yücel. ${ }^{1}$ Physical Medicine and Rehabilitation; ${ }^{2}$ Radiology, Kocatepe University, Afyon, Turkey

\subsection{6/annrheumdis-2001.545}

Objectives Low back pain (LBP) is a major health problem affecting the adult population. Postural changes are one of the risk factor in some cases. Abnormal posture makes a strain on ligaments and muscules that indirectly affects the curvature of lumbal spine. The purpose of this study was to investigate the relationship between the angles of lumbar spine and spinal mobility in acute and chronic LBP patients.

Methods Fifty patients (25 female, 25 male) with chronic Low Back Pain (CLBP) and fifty patients (30 female, 20 male) with acute low back pain (ALBP) were included the study. Both groups were subjected to lumbosacral radiography in lateral position while they were standing. Measurements were made directly from the radiographs. Patients were assessed by parameters such as spinal mobility, sacral inclination angle, lumbosacral angle and sacral horizontal angle. Spinal mobility included lumbar spinal flexion and extension and measured by double inclinometer method.

Results There were no statistical differences between age and gender in both groups $(\mathrm{p}>0.05)$. The value of the SIA was significantly increased in CLBP patients compared to ALBP group. It was also correlated with lumbar extension in CLBP patients ( $p$ $<0.005)$. There was no statistical difference in LSA between ALBP and CLBP groups $(p>0.05)$. There was also no observable difference between two groups and no correlation with lomber flexion and extension for men and women $(\mathrm{p}>0.05)$.

Conclusion The weakness of these muscles may cause a forward posture and an increase in lumbar lordosis. Additional researches should be helpful to confirm the relationship between the angles of lumbar spine and low back pain.

\section{REFERENCES}

1 Amonoo-Kuofi HS. Changes in the lumbosacral angle, sacral inclination and the curvature of the lumbar spine during aging. Acta Anat. 1992;145:373-7

2 Youdas JW, Garrett TR, Harmsen S, et al. Lumbar lordosis and pelvic inclination of asymptomatic adults. Phys Ther. 1996;76:1066-81

3 Youdas JW, Garrett TR, Egan KS, Therneau TM. Lumbar lordosis and pelvic inclination in adults with chronic low back pain. Phys Ther. 2000;80:261-75

4 Hansson $T$, Bigos $S$, Beecher $P$, Wortley $M$. The lumbar lordosis in acute and chronic low-back pain. Spine 1985;10(2):154-5

\section{FRI0242 A LARGE MULTICENTRE COMPARATIVE EFFICACY, TOLERABILITY AND QUALITY OF LIFE TRIAL BETWEEN TTS FENTANYL AND SUSTAINED RELEASE MORPHINE, IN STRONG-OPIOID NAIIVE PATIENTS WITH CHRONIC LOW BACK PAIN}

L Allan, L Tooke. The Pain Clinic, Northwick Park Hospital, Harrow, UK

10.1136/annrheumdis-2001.546

Background Low back pain is a common condition affecting approximately $80 \%$ of the population during their lifetime. ${ }^{1}$ It is not only a leading cause of disability in developed countries, but is also the most costly biopsychosocial chronic pain condition in 